\title{
Performance of Multihop Latency Aware Scheduling in Delay Constrained Ad Hoc Networks
}

\author{
Ben Liang \\ Department of Electrical and Computer Engineering \\ University of Toronto, Toronto, Ontario, M5S 3G4, Canada \\ Email: liang@comm.utoronto.ca
}

\begin{abstract}
This paper addresses the problem of optimizing packet transmission schedule at the Medium Access Control layer of an ad hoc network, in order to minimize the probability of packet loss due to excessive end-to-end delay. We study a family of Multihop Latency Aware (MLA) schedules, where the scheduling of each packet takes into account its remaining hop count and remaining lifetime. We propose a numerical analysis framework to evaluate the performance of MLA scheduling. Using the proposed analysis framework, we study the optimization of MLA parameters to minimize packet loss probability. We show that the MLA scheme significantly out performs other scheduling schemes such as First-In-First-Out, Earliest-Deadline-First, and Largest-Distance-First.
\end{abstract}

\section{INTRODUCTION}

As multimedia applications become more prevalent in wireless networks, to ensure the reliable transmission of multimedia data across multiple hops of wireless links remains a challenging problem[10]. In particular, an important criterion for the successful decoding of multimedia streams is the end-to-end transmission delay of packets[16]. Typically, if a multimedia streaming receiver has not completely received an audio/video frame by its pre-defined playback deadline, the frame is obsolete. A small probability of frame loss is required for acceptable perceived audio/video quality. Thus, multimedia communication poses stringent requirements on the delay threshold of packet transmission.

For multimedia applications in ad hoc networks, a major contribution to the overall transmission delay is at the Medium Access Control (MAC) layer, due to distributed multi-node contention over radio channels. However, the most commonly employed MAC layer protocols for ad hoc networks[10] are not designed based on the multihop end-to-end delay of a packet. Examples of these protocols include RI-BTMA[28], MACA[15], MACAW[4], FAMA[8], IEEE 802.11 DCF[5], and DBTMA[11]. All of them mainly concern the packet transmission within a confined local neighborhood, without considering the accumulated effects over the entire route traversed by a packet. These protocols follow the traditional layering approach[17], which provide solutions to the MAC problem independently of the other protocol layers.

To support multimedia applications, the efficient operation of ad hoc networks requires flexibility and adaptation across the entire protocol stack. Recent empirical data have shown that the performance of one protocol layer in an ad hoc network is closely related to the time-varying characteristics of the network at the other layers[3][27]. Therefore, the cross-layer approach to ad hoc network design, which allows the jointly adaptive optimization of the protocol layers, is important to the successful deployment of future large-scale multimedia ad hoc networks.

In this paper, we consider the problem of optimizing the packet transmission schedule at the MAC layer of an ad hoc network, through cross-layer information exchange. The goal is to minimize the probability of packet loss due to excessive end-to-end delay. We propose and evaluate the performance of a family of Multihop Latency Aware (MLA) schedules, where the transmission order of packets depends on both their remaining hop count and their remaining lifetime. We provide a numerical framework to evaluate the performance of MLA scheduling and study the optimization of packet ordering under different system parameters.

The rest of this paper is organized as follows. In Section II, we give detailed description of the MLA scheduling scheme. In Section III, we summarize the related work. In Section IV, we provide a numerical analysis framework for computing the probability of packet loss. In Section $\mathrm{V}$, we study the performance of the MLA scheduling scheme and its optimization given various system parameters. Finally, Section VI concludes.

\section{Multihop Latency Aware Scheduling}

We consider a cross-layer ad hoc network design where the MAC layer is aware of the routing hop count and application lifetime of contending packets. In the MLA design, the scheduling of each packet takes into account the number of remaining hops, denoted $H$, from the the current location of the packet to the its destination, and the remaining lifetime, denoted $T$, of the packet.

Within each node, the values of $H$ and $T$ of all packets can be made available to the MAC layer through crosslayer information exchange. The hop count information is recorded in most of the routing protocols proposed for ad hoc networks[10][25]. For example, all link-state, distance-vector, and source-routing protocols require that a node stores the exact hop count from itself to all active destination nodes[19]. Each packet is assigned an expiry time based on the delay threshold 
set by the application[16]. We assume that this information is embedded within each packet, so that a node can compare this value with the current time to compute the remaining lifetime of the packet.

All packets within a contention area compete for access to the shared medium. The nodes within a contention area can exchange the $H$ and $T$ values of the contending packets through short control messages. These control messages may be piggybacked within the previously transmitted data packets[2], and, in the case of IEEE 802.11 compliant protocols, within the RTS/CTS/ACK conrol pakcets[14].

Clearly, the less remaining lifetime a packet has, and the more hops it has to traverse, the more urgent the packet is. Therefore, based on the values of $H$ and $T$, an MLA packet ranking function is defined as

$$
\gamma(H, T)=\frac{T^{\alpha}}{H},
$$

where $\alpha$ can take any non-negative value. Assuming ideal scheduler implementation, the packet with the smallest $\gamma(H, T)$ among a group of contending packets is transmitted first.

Note that (1) represents a family of ranking functions that also include any function of the form $\frac{T^{a}}{H^{b}}$. To see this, we let $\alpha=\frac{a}{b}$. Then, it is clear that, given any $\left(H_{1}, T_{1}\right)$ and $\left(H_{2}, T_{2}\right)$ such that $\frac{T_{1}{ }^{a}}{H_{1}{ }^{b}}<\frac{T_{2}{ }^{a}}{H_{2} b}$, we have $\gamma\left(H_{1}, T_{1}\right)<\gamma\left(H_{2}, T_{2}\right)$, i.e., $\gamma(H, T)$ gives the same ordering of packets as $\frac{T^{a}}{H^{b}}$ does. Therefore, $\alpha$ can be viewed as a factor that represents the relative weight of the remaining hop count and the remaining lifetime in ranking the urgency of packets. In this work, we study the optimization of $\alpha$ under different system parameters.

\section{RELATED WORK}

Much existing work on distributed scheduling in the ad hoc networking environment concentrates on ensuring fair access to the shared medium[2][23][21][22][26][13]. However, there is comparatively less work on scheduling to ensure end-to-end delay for ad hoc networks.

The references [2][14] provide solutions to implementing distributed multiple-access schemes that approximate a given schedule. These methods do not directly concern the end-to-end delay guarantee. However, they can be employed to implement the MLA scheduling scheme.

In [13], a priority index based multihop coordinated scheduling scheme is proposed for ad hoc networks, with three types of index assignments. In particular, the Time To Live assignment scheme is the special case of MLA with $\alpha=\infty$. The Uniform Delay Budget is similar to the case of MLA with $\alpha=1$, but it only considers the average lifetime allowance per hop, instead of the remaining lifetime as in MLA. Furthermore, the performance analysis in [14] neglects the queueing delay by assuming that there is only one packet in each node's transmission queue. We do not make this assumption in this work.

Multihop scheduling has been considered in wireline networks to ensure end-to-end Quality-of-Service bounds[20][17][6][1][18]. The most commonly studied scheduling schemes are First-In-First-Out (FIFO), where the first packet to enter a transmission queue is given the highest priority, Global Earliest-Deadline-First (EDF), where the packet with the least remaining lifetime is given the highest rank, and Longest-Distance-First (LDF), where the packet with the highest remaining hop count is given the highest rank.

The EDF and LDF schemes are the same as the special cases of MLA with $\alpha=\infty$ and $\alpha=0$, respectively. In this work, we consider general values of $\alpha$, and give a numerical framework for choosing the optimal values of $\alpha$ under different system parameters.

In addition, studies have shown that EDF significantly out performs FIFO in general. Therefore, in this work, we will compare the performance of MLA with only EDF and LDF.

\section{Performance Analysis of MLA Scheduling}

In this section, we present an iterative numerical framework to evaluate the performance of the MLA scheduling scheme. We are particularly interested in computing $p_{\text {loss }}$, the overall probability of packet loss due to insufficient remaining lifetime.

\section{A. Network Model}

In the source node, a multimedia application sends out data packets encoded with packet expiry time. The packet is processed by the network layer, encapsulating it with routing information, including the hop count to its destination node. For a packet entering the transmission queue of the source node, we denote its initial hop count $H_{0}$ and initial lifetime $T_{0}$, with a general distribution $P_{H_{0} T_{0}}(h, t)$.

The packet is then forwarded successively along the nodes within the predefined route, where its remaining hop count $H$ and remaining lifetime $T$ are updated. At each hop along this route, the packet's ranking function $\gamma(H, T)$, as defined in (1), is compared with all other contending packets. The packet with the highest rank (i.e., smallest $\gamma(H, T)$ ) is scheduled to be transmitted immediately. Ties are broken randomly. We assume that all packets have the same length and that the transmission time of each packet is constant. Throughout this paper, we normalize all time durations such that the transmission time represents 1 time unit.

A node periodically inspects the remaining life time of all packets within its transmission queue. The node discards any packet that cannot arrive at its destination before its expiry time. This includes all packets that have $T<H$. Note that, since a packet's remaining lifetime decreases as the packet waits in the transmission queue, a packet can be expunged during its waiting time, even though it had adequate residual lifetime when it first arrived.

In this work, we only consider networks where the amount of discarded packets is small. For highly congested networks, so that many packets are dropped, a selective admission control protocol is necessary, instead of attempting to transmit all packets. The optimal combination of the MLA scheduling 
scheme and an admission control protocol is outside the scope of this paper.

In addition, we assume that packet arrivals into a contention area can be approximated by a Poisson stream with arrival rate $\lambda$. The value of $\lambda$ can be readily measured or estimated in practice. The Poisson approximation is well justified, due to the random delays incurred under multiple-node contention, and the fact that the transmitted packet of a contention area is randomly forwarded to one of the nodes within the area.

The proposed iterative analysis framework is divided into three steps as shown in the following subsections.

\section{B. Computing Packet Delay Distribution Given $P_{H_{e} T_{e}}(h, t)$}

Let $H_{e}$ and $T_{e}$ represent the remaining hop count and the remaining lifetime of a packet when it enters the transmission queue of a source or intermediate node. Let $P_{H_{e} T_{e}}(h, t)$ be their joint probability function, which can be computed as shown in the next subsection. For now, we suppose it is given.

Let $R(h, t)$ be the amount of delay that a packet experiences at a node, given that the packet enters the node with $H_{e}=h$ and $T_{e}=t$. Let $P_{R(h, t)}(r)$ be its probability function. We further allow the notation $P_{R(h, t)}(\infty)$ to represent the probability that the packet is discarded.

To compute $P_{R(h, t)}(r)$, we first need to determine $p_{x}(h, t)$, the probability that a packet with $h$ remaining hops and $t$ remaining lifetime is transmitted at the current time step. Let $N$ be the number of packets waiting to be transmitted within the contention area. Let $P_{N}(n)$ be its probability function and $P_{N}{ }^{*}(z)$ its probability generating function. When the packet transmission time is constant, as a special case of the PollaczekKhintchine formula [9], we have

$$
P_{N}^{*}(z)=\frac{(1-\lambda)(1-z)}{1-z e^{\lambda(1-z)}}
$$

where $\lambda$ is the rate of packet arrival, and the time to transmit a packet is normalized to 1 . Here, we have assumed that the amount of discarded packets is small, as explained in Section IV-A.

Given a packet of rank $\gamma(h, t)$, let $M$ and $U$ be the number of packets within the contention area that has greater rank and equal rank, respectively. The packet is transmitted at the current time step if $M=0$ and the packet is chosen among all $U+1$ packets of the same rank. Therefore, the probability that the packet is transmitted at the current time step is

$$
\begin{aligned}
p_{x}(h, t) & =\operatorname{Pr}\{M=0\} \sum_{u=0}^{\infty} P_{N \mid M=0}(u) \frac{1}{u+1} \\
& =\sum_{u=0}^{\infty} \operatorname{Pr}\{M=0, U=u\} \frac{1}{u+1}
\end{aligned}
$$

Furthermore, it can be shown that the $z$ transform of $\operatorname{Pr}\{M=0, U=u\}$, taken over $u$ is

$$
\begin{aligned}
& P_{N}^{*}\left[1-p_{g}(h, t)-p_{e}(h, t)(1-z)\right] \\
= & \frac{(1-\lambda)\left[p_{g}(h, t)+p_{e}(h, t)(1-z)\right]}{1-\left[1-p_{g}(h, t)-p_{e}(h, t)(1-z)\right] e^{\lambda\left[p_{g}(h, t)+p_{e}(h, t)(1-z)\right]}},
\end{aligned}
$$

where $p_{g}(h, t)$ and $p_{e}(h, t)$ are the probabilities that a packet within the contention area has rank higher than $\gamma(h, t)$ and equal to $\gamma(h, t)$, respectively, i.e.,

$$
\begin{aligned}
& p_{g}(h, t)=\sum_{\gamma\left(h^{\prime}, t^{\prime}\right)<\gamma(h, t)} P_{H_{q} T_{q}}\left(h^{\prime}, t^{\prime}\right) \\
& p_{e}(h, t)=\sum_{\gamma\left(h^{\prime}, t^{\prime}\right)=\gamma(h, t)} P_{H_{q} T_{q}}\left(h^{\prime}, t^{\prime}\right),
\end{aligned}
$$

where $P_{H_{q} T_{q}}(h, t)$ is the steady-state distribution of the remaining hop count and lifetime of a packet waiting to be transmitted within the contention area. Detailed derivation of the above is omitted due to page limitation.

Thus, we have

$$
\begin{aligned}
& p_{x}(h, t)= \\
& \sum_{u=0}^{\infty} \mathcal{Z}^{-1}\left\{P_{N}{ }^{*}\left[1-p_{g}(h, t)-p_{e}(h, t)(1-z)\right]\right\} \frac{1}{u+1},
\end{aligned}
$$

where $\mathcal{Z}^{-1}$ denotes inverse $z$-transform. In general, the above inverse $z$-transform does not have a closed from solution. In this work, we compute its numerical solution by inverse discrete Fourier transform.

Next, we present an iterative algorithm to compute $P_{H_{q} T_{q}}(h, t)$. Given the distribution function $P_{H_{e} T_{e}}(h, t)$ and the probabilities $p_{x}(h, t)$, we can construct a Markov chain whose states consist all pairs of $(h, t)$, each representing the remaining hop count and remaining lifetime of a packet within the contention area. If a packet is in state $(h, t)$ with $t \geq h+1$, with probability $1-p_{x}(h, t)$, it will not be transmitted in the current time step, and hence it will transit to new state $(h, t-1)$. Since, in equilibrium, the rate of packets entering the system equals the rate of packets leaving the system, with probability $p_{x}(h, t)$, the packet is transmitted and re-enters the system with state probability distribution ${ }^{1} P_{H_{e} T_{e}}(h, t)$. When a packet is in a state where $t=h$, if it is not transmitted immediately, it will be discarded since it has zero probability of arriving at the destination before its lifetime expires. Therefore, such packets always leaves the system in the next step, whether it is transmitted or not. Thus, the transition probabilities of this Markov chain can be summarized as follows:

$$
\begin{aligned}
& \operatorname{Pr}\left\{(h, t) \rightarrow\left(h^{\prime}, t^{\prime}\right)\right\}= \\
& \left\{\begin{array}{cc}
1-p_{x}(h, t)+p_{x}(h, t) P_{H_{e} T_{e}}(h, t-1) \\
\quad t \geq h+1, h^{\prime}=h, t^{\prime}=t-1 \\
p_{x}(h, t) P_{H_{e} T_{e}}\left(h^{\prime}, t^{\prime}\right), & t \geq h+1,\left(h^{\prime}, t^{\prime}\right) \neq(h, t) \\
P_{H_{e} T_{e}}\left(h^{\prime}, t^{\prime}\right), & t=h .
\end{array}\right.
\end{aligned}
$$

Let $\mathbf{P}_{q}$ be the transition probability matrix of this Markov chain. Let $\mathbf{p}_{q}$ be the vector version of $P_{H_{q} T_{q}}(h, t)$. We need to solve $\mathbf{p}_{q}=\mathbf{p}_{q} \mathbf{P}_{q}$ for the steady-state distribution of $P_{H_{q} T_{q}}(h, t)$. However, as indicated in $(2), p_{x}(h, t)$ depends on

\footnotetext{
${ }^{1}$ Alternatively, we can create a new reservoir state $(0,0)$. All packets leaving the system go into $(0,0)$. While in $(0,0)$, the Markov chain next transits into $(h, t)$ with probability distribution $P_{H_{e} T_{e}}(h, t)$. Clearly, this construction yields the same solution.
} 
$\mathbf{p}_{q}$, and, hence, $\mathbf{P}_{q}$ is a function of $\mathbf{p}_{q}$. Therefore, the standard Markov chain steady-state analysis does not hold. Instead, the follow iterative algorithm on $P_{H_{q} T_{q}}{ }^{(i)}(h, t)$ can be use:

Initialization: $P_{H_{q} T_{q}}{ }^{(0)}(h, t)=P_{H_{e} T_{e}}(h, t)$
Step 1: Compute $p_{x}{ }^{(i)}(h, t)$ following (2)-(3)
Step 2: Construct $\mathbf{P}_{q}{ }^{(i)}$ following (4)
Step 3: Let $\mathbf{p}_{q}{ }^{(i+1)}=\mathbf{p}_{q}{ }^{(i)} \mathbf{P}_{q}{ }^{(i)}$
Repeat from Step 1

By the Markov chain construction in (4), ignoring the singleton closed sets $\{(h, t) \mid t<h\}$, the stochastic matrix $\mathbf{P}_{q}{ }^{(i)}$ is scrambling $^{2}$ for all $i$. Therefore, the sequence $\left\{\mathbf{P}_{q}{ }^{(i)}\right\}$ is ergodic, and it can be shown that the above iteration converges[7].

In the above iteration, $p_{x}{ }^{(i)}(h, t)$ converges to $p_{x}(h, t)$. We can then compute $P_{R(h, t)}(r)$ with the following recursion:

$$
P_{R(h, t)}(1)=p_{x}(h, t),
$$

and, for $2 \leq r \leq t-h+1$,

$$
P_{R(h, t)}(r)=\left[1-\sum_{i=1}^{r-1} P_{R(h, t)}(i)\right] p_{x}(h, t-r+1) .
$$

Finally,

$$
P_{R(h, t)}(\infty)=1-\sum_{i=1}^{t-h+1} P_{R(h, t)}(i)
$$

\section{Computing the Steady-State Distribution $P_{H_{e} T_{e}}(h, t)$}

The previous section gives a method to determine $P_{R(h, t)}(r)$ given $P_{H_{e} T_{e}}(h, t)$. Next, we show that this can be used to in turn compute $P_{H_{e} T_{e}}(h, t)$.

Given the initial hop-count and lifetime distribution of a packet, $P_{H_{0} T_{0}}(h, t)$, and the delay distribution, $P_{R(h, t)}(r)$, we can construct a Markov chain whose states consist all pairs of $(h, t)$, each representing the remaining hop count and remain lifetime of a packet when it enters a new node. Suppose the state of a packet when it enters the current node is $(h, t)$, where $h>1$. With probability $P_{R(h, t)}(r)$, for all $1 \leq r \leq$ $t-h+1$, the packet will be transmitted after $r$ time units, and hence, will enter the next node with state $(h-1, t-r)$. With probability $P_{R(h, t)}(\infty)$, however, the packet will be discarded. In equilibrium, the rate of packets entering the system equals the rate of packets leaving the system. Therefore, with probability $P_{R(h, t)}(\infty)$, the Markov chain transits to a new state with probability distribution ${ }^{3} P_{H_{0} T_{0}}(h, t)$. When a packet enters the node immediately in front of its destination node, it is in state $(1, t)$. It is either discarded with probability $P_{R(1, t)}(\infty)$ or successfully transmitted to the destination node with probability $1-P_{R(1, t)}(\infty)$. In either case, the packet will leave the system in the next state transition. Therefore, the

\footnotetext{
${ }^{2}$ A stochastic matrix is scrambling[12] if for any two states $i$ and $j$, there exists a state $k$, such that $k$ is reachable from both $i$ and $j$.

${ }^{3}$ This is equivalent to an alternative construction using a reservoir state. See Footnote 1 for a similar case.
}

transition probabilities of this Markov chain can be summarized as follows:

$$
\begin{aligned}
& \operatorname{Pr}\left\{(h, t) \rightarrow\left(h^{\prime}, t^{\prime}\right)\right\}= \\
& \left\{\begin{array}{c}
P_{R(h, t)}(r)+P_{R(h, t)}(\infty) P_{H_{0} T_{0}}\left(h^{\prime}, t^{\prime}\right), \\
h>1, t \leq h, h^{\prime}=h-1, t^{\prime}=t-r, 1 \leq r \leq t-h+1 \\
P_{R(h, t)}(\infty) P_{H_{0} T_{0}}\left(h^{\prime}, t^{\prime}\right), \quad h \leq 1, t \leq h, h^{\prime} \neq h-1 \\
P_{H_{0} T_{0}}\left(h^{\prime}, t^{\prime}\right), \\
h=1 .
\end{array}\right.
\end{aligned}
$$

Let $\mathbf{P}_{e}$ be the transition probability matrix of this Markov chain. Let $\mathbf{p}_{e}$ be the vector version of $P_{H_{e} T_{e}}(h, t)$. We need to solve $\mathbf{p}_{e}=\mathbf{p}_{e} \mathbf{P}_{e}$ for the steady-state distribution of $P_{H_{e} T_{e}}(h, t)$. However, from the last section, it is clear that $P_{R(h, t)}(r)$ depends on $\mathbf{p}_{e}$ and, hence, $\mathbf{P}_{e}$ is a function of $\mathbf{p}_{e}$. Therefore, the standard Markov chain steady-state analysis does not hold. Instead, the follow iterative algorithm on $P_{H_{e} T_{e}}{ }^{(i)}(h, t)$ can be use:

$$
\begin{aligned}
& \text { Initialization: } P_{H_{e} T_{e}}{ }^{(0)}(h, t)=P_{H_{0} T_{0}}(h, t) \\
& \text { Step 1: Compute } P_{R(h, t)}(r) \text { as in Section IV-B } \\
& \text { Step 2: Construct } \mathbf{P}_{e}{ }^{(i)} \text { following (5) } \\
& \text { Step 3: Let } \mathbf{p}_{e}{ }^{(i+1)}=\mathbf{p}_{e}{ }^{(i)} \mathbf{P}_{e}{ }^{(i)} \\
& \text { Repeat from Step 1 }
\end{aligned}
$$

Similar to the computation in Section IV-B, here we can show that the stochastic matrix $\mathbf{P}_{e}{ }^{(i)}$ is scrambling, and the above iteration converges. When convergence is reached, we obtain the steady-state distribution $P_{H_{e} T_{e}}(h, t)$ and the corresponding $P_{R(h, t)}(r)$.

\section{Probability of Packet Loss}

After the values of $P_{R(h, t)}(r)$ are computed, the probability of packet loss can be determined using a Markov chain similar to (5). In addition to the states $(h, t)$, representing the remaining hop count and remaining lifetime of a packet when it enters a node, we introduce two absorbing states success and loss, which represent the cases of a packet being successfully transmitted to the destination and being lost due to insufficient remaining lifetime, respectively. When a packet arrives at its destination or is discarded, instead of modeling its re-entry the system, the Markov chain goes into one of the absorbing states. Hence, the new Markov chain is expressed by

$$
\left\{\begin{array}{l}
\operatorname{Pr}\left\{(h, t) \rightarrow\left(h-1, t^{\prime}\right)\right\}=P_{R(h, t)}(r), \\
\quad h>1, t \leq h, t^{\prime}=t-r, 1 \leq r \leq t-h+1 \\
\operatorname{Pr}\{(h, t) \rightarrow \text { loss }\}=P_{R(h, t)}(\infty), \quad t \leq h \\
\operatorname{Pr}\{(1, t) \rightarrow \text { success }\}=1-P_{R(1, t)}(\infty) .
\end{array}\right.
$$

Since the values of $P_{R(h, t)}(r)$ are already given, Equation (6) represents a homogeneous Markov chain. Therefore, standard techniques can be applied to compute the absorption probabilities of this chain[24]. In particular, let $\mathbf{P}_{s}$ be the transient part of the transition probability matrix of the Markov chain. Let $p_{s}(h, t)$ be the probability that a packet in state $(h, t)$ is 


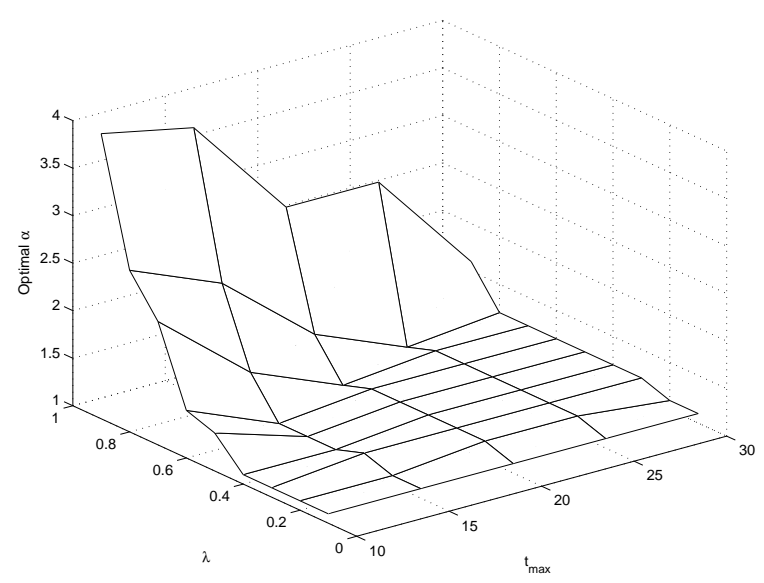

Fig. 1. Optimal $\alpha$, for different values of $t_{\max }$ and $\lambda$.

eventually successfully transmitted to its destination (absorbed into the success state), and let $\mathbf{p}_{s}$ be its vector version. Let $p_{a}(h, t)=\operatorname{Pr}\{(h, t) \rightarrow$ success $\}$ be the probability that a packet in state $(h, t)$ is transmitted to its destination in the next step, and let $\mathbf{p}_{a}$ be its vector version. It can be shown that

$$
\mathbf{p}_{s}=\left(I-\mathbf{P}_{s}\right)^{-1} \mathbf{p}_{a},
$$

where $I$ denotes the identity matrix.

Finally, the overall packet loss probability, given the initial packet hop-count and lifetime distribution $P_{H_{0} T_{0}}(h, t)$ is given by

$$
p_{\text {loss }}=\sum_{h, t} P_{H_{0} T_{0}}(h, t)\left[1-p_{s}(h, t)\right] .
$$

\section{Numerical Analysis And Simulation}

In this section, we use the proposed numerical analysis framework to study the performance of MLA scheduling given various system parameters.

\section{A. Optimality of $\alpha$}

We are interested in the probability of packet loss with different ranking functions $\gamma(h, t)=T^{\alpha} / H$, for different values of $\alpha$. We consider the cases where the initial hop count of a packet is uniformly distributed between 1 and $h_{\max }$. In particular, we set $h_{\max }=10$. Given any source and destination node pair, the initial lifetime of a packet is randomly set such that it has uniform distribution between the hop count and $t_{\max }$. For each set of system parameters, we compute the probability of packet loss for a series of $\alpha$ values, ranging from 0.1 to 4. The optimal values of $\alpha$, denoted, $\alpha_{\text {opt }}$, for different set of values of $t_{\max }$ and $\lambda$, which minimizes the probability of packet loss, are presented in Figure 1. In these plots, $t_{\max }$ ranges from 10 to 30 , and $\lambda$ ranges from 0.1 to 0.9 .

Figure 1 indicates that the optimal $\alpha$ increases as the traffic load increases, and it decreases as the initial packet lifetime increases. For example, with $t_{\max }=15$, when $\lambda=0.4$, we have $\alpha_{\text {opt }}=1.2$, and when the traffic load is increased to $\lambda=$ 0.8 , we have $\alpha_{\text {opt }}=2.4$. With $\lambda=0.5$, when $t_{\max }=10$, we

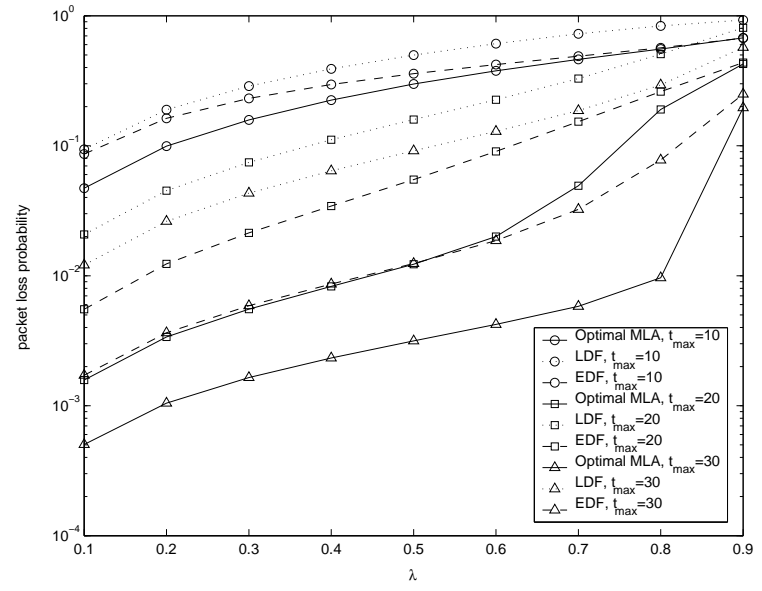

Fig. 2. Probability of packet loss vs. $\lambda$, comparing MLA with optimal $\alpha$ against $\mathrm{LDF}$ and $\mathrm{EDF}$.

have $\alpha_{\text {opt }}=1.5$, and when the initial packet lifetime increases such that $t_{\max }=20$, we have $\alpha_{\text {opt }}=1.3$.

The value of $\alpha$ represents the relative weight of the remaining hop count and the remaining lifetime in ranking the urgency of packets. The above suggests that the remaining hop count is a more important factor (i.e., $\alpha$ should be small) when the network is lightly loaded and when packets have long lifetimes. Likewise, the remaining lifetime is a more important factor (i.e., $\alpha$ should be large) when the network is heavily loaded and when packets have short lifetimes. In particular, in the extreme case where the networks is highly stressed, $\alpha_{o p t}=\infty$ (i.e., the EDF scheduling scheme) should be used. This matches the observed optimality of EDF in previous literature.

We note that, for a wide range of system parameters, the optimal $\alpha$ is between 1.2 and 1.5 .

\section{B. Performance of Optimal MLA}

The following figures demonstrate the performance gain of using the optimal $\alpha$ values in MLA, against the conventional LDF and EDF schemes, where either only the remaining hop count or only the remaining lifetime is used in ranking packets. We have omitted the comparison results with the FIFO scheme, since our results agree with existing literature in concluding that EDF significantly out performs FIFO.

Figures 2 and 3 present the probability of packet loss for MLA, with optimal $\alpha$, and compare it with those of the LDF and EDF schemes. In both figures, the distributions of packet initial hop count and initial lifetime are the same as in Section $\mathrm{V}-\mathrm{A}$. The same ranges of values of $\lambda$ and $t_{\max }$ as in Section $\mathrm{V}-\mathrm{A}$ are used, but only the data points for $t_{\max }=10,20,30$ and $\lambda=0.2,0.4$ are shown.

These figures demonstrate that the optimized MLA scheduling always out performs LDF and EDF. In general, the performance gain decreases quickly as the network traffic load, represented by $\lambda$, increases, but it decreases slowly as the packet lifetime, represented by $t_{\max }$, decreases. This matches the observations made in Section V-A. 


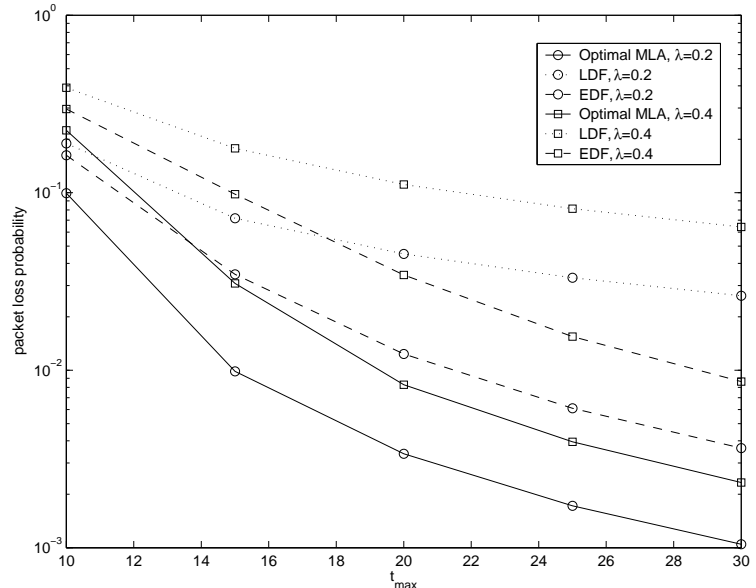

Fig. 3. Probability of packet loss vs. $t_{\max }$, comparing MLA with optimal $\alpha$ against LDF and EDF.

For a wide range of system parameters, the performance gain is significant. For example, in the cases where $t_{\max }=20$ and $\lambda=0.5$, the probabilities of packet loss in LDF, EDF, and the optimal MLA, are $0.16,0.055$, and 0.012 , respectively. In this case, the number of loss packets in the optimal MLA are $22 \%$ of the number of loss packets in EDF and only $7.5 \%$ of the number of loss packets in LDF.

\section{CONCLUSIONS}

End-to-end delay is one of the most important service criteria for multimedia applications. In this paper, we consider the problem of improving the packet transmission schedule at the MAC layer of an ad hoc network, in order to minimize the probability of packet loss due to excessive end-to-end delay. We present a Multihop Latency Aware scheduling scheme, which can significantly improve the delay performance of multimedia data transmission in ad hoc networks.

We propose a numerical analysis framework for evaluating the performance of MLA scheduling. Using the proposed analysis framework, we study the optimal MLA parameter $\alpha$ that minimizes the packet loss probability. Our numerical results show that the remaining hop count is a more important factor (i.e., $\alpha$ should be small) when the network is lightly loaded and when packets have long lifetimes. Likewise, the remaining lifetime is a more important factor (i.e., $\alpha$ should be large) when the network is heavily loaded and when packets have short lifetimes. We also observe that, for a wide range of system parameters, the optimal value of $\alpha$ is between 1.2 and 1.5. We further show that, with an optimal value of $\alpha$, the MLA scheme can significantly out perform other common scheduling schemes such as EDF and LDF.

\section{REFERENCES}

[1] M. Andrews and L. Zhang, "Minimizing end-to-end delay in high-speed networks with a simple coordinated schedule," Proc. IEEE INFOCOM, March 1999.
[2] C. Barrack and K-Y. Siu, "A distributed scheduling algorithm for quality of service support in multiaccess networks," Proc. of IEEE ICNP, October 1999.

[3] C. Barrett, M. Drozda, A. Marathe, and M. V. Marathe, "Characterizing the interaction between routing and MAC protocols in ad-hoc networks," Proc. ACM MOBIHOC, pp. 92-103, June 2002.

[4] V. Bharghavan, A. Demers, S. Shenker, and L. Zhang, "MACAW: A media access protocol for wireless LAN's," Proc. ACM SIGCOMM, pp.212-225, 1994

[5] G. Bianchi, "Performance Analysis of the IEEE 802.11 Distributed Coordination Function," IEEE J. Selected Areas in Communications, vol. 18, no. 3, pp. 535-547, March 2000.

[6] F. M. Chiussi and V. Sivaraman, "Achieving high utilization in guaranteed services networks using early-deadline-first scheduling," Proc. IEEE IWQoS, 1998.

[7] I. Daubechies and J. C. Lagarias, "Sets of matrices all infinite products of which converge," Linear Algebra and Its Applications, vol. 161, pp. 227-263, 1992.

[8] C.L. Fullmer, J.J. Garcia-Luna-Aceves, "Floor acquisition multiple access (FAMA) for packet-radio networks," Proc. ACM SIGCOMM, pp.262-273, 1995.

[9] D. Gross and C. M. Harris, Fundamentals of Queueing Theory, 3rd ed., John Wiley \& Sons, 1998.

[10] Z. J. Haas, J. Deng, B. Liang, P. Papadimitratos, and S. Sajama, "Wireless Ad Hoc Networks," John G. Proakis ed., Wiley Encyclopedia of Telecommunications, John Wiley \& Sons, 2002.

[11] Z. J. Haas and J. Deng, "Dual Busy Tone Multiple Access (DBTMA) a multiple access control scheme for ad hoc networks," IEEE Trans. on Communications, vol. 50, no. 6, pp. 975-985, June 2002.

[12] J. Hajnal, "Weak ergodicity in nonhomogeneous Markov chains," Proc. Cambridge Philosophical Society, vol. 54, pp. 233-246, 1958.

[13] V. Kanodia, C. Li, A. Sabharwal, B. Sadeghi, and E. Knightly, "Distributed multi-hop scheduling and medium access with delay and throughput constraints," Proc. ACM MOBICOM, pp. 200-209, 2001.

[14] V. Kanodia, C. Li, A. Sabharwal, B. Sadeghi, and E. Knightly, "Ordered packet scheduling in wireless ad hoc networks: mechanisms and performance analysis," Proc. ACM MOBIHOC, 2002.

[15] P. Karn, "MACA - A new channel access method for packet radio," ARRL/CRRL Amateur Radio 9th Computer Networking Conference, pp.134-140, 1990.

[16] G. Karlsson, "Asynchronous transfer of video," IEEE Communications Mag., vol. 34, pp. 106-113, February 1996.

[17] S. Keshave, An Engineering Approach to Computer Networking, AddisonWesley, 1997.

[18] C. Li and E. W. Knightly, " Coordinated multihop scheduling: a framework for end-to-end services," IEEE/ACM Trans. Networking, vol. 10, no. 6, December 2002.

[19] B. Liang and Z. J. Haas, "Optimizing route-cache lifetime in ad hoc networks," Proc. IEEE INFOCOM, April 2003.

[20] J. Liebeherr, D. E. Wrege, and D. Ferrari, "Exact admission control for networks with a bounded delay service," IEEE/ACM Trans. Network, vol. 4, no. 6, December 1996.

[21] H. Luo, S. Lu, and V. Bharghavan, "A new model for packet scheduling in multihop wireless networks," Proc. ACM MOBICOM, August, 2000.

[22] T. Nandagopal, T. Kim, X. Gao, and V. Bharghavan, "Achieving MAC layer fairness in wireless packet networks," Proc. ACM MOBICOM, August, 2000.

[23] T. Ozugur, M. Naghshineh, P. Kermani, and J. Copeland, "Fair media access for wireless LANs," Proc. IEEE Globecom, 1999.

[24] A. Papoulis and S. U. Pillai, Probability, Random Variables, and Stochastic Processes, Fourth Edition, McGraw-Hill, 2002.

[25] C. E. Perkins, ed., Ad Hoc Networking, Addison-Wesley Longman, 2001.

[26] N. Vaidya, P. Bahl, and S. Gupta, "Distributed fair scheduling in a wireless LAN," Proc. ACM MOBICOM, August 2000.

[27] K. K. Vadde and V. R. Syrotiuk, "Factor Interaction on Service Delivery in Mobile Ad Hoc Networks," IEEE J. Selected Areas in Communications, vol. 22, no. 7, pp. 1335-1346, September 2004.

[28] C. Wu and V. O. K. Li, "Receiver-initiated busy-tone multiple access in packet radio networks," Proc. ACM SIGCOMM, pp.336-342, 1987. 